\title{
II. Usages du cannabis indica dans le traitement des névroses et dyspepsies gastriques.
} Anwendung der Cannabis indica in der Behandlung der Neurosen und gastrischen Dyspepsieen. Par G. Sée, Paris.

\section{Premiere partie.}

Pharmacologie.

Nons diviserons ce travail en trois parties: la première sera consacrée à la pharmacologie clu cannabis; la deuxième, à l'étude sommaire des névroses et dyspepsies gastriques; la troisieme, aux applications du cannabis dans cesdiverscas.

Le cannabis sativa (Urticariées) est une plante provenant des Indes occidentales, qui est cultivée en Europe à cause de ses graines très oléagineuses. Une variété de cette plante, le cannabis sativa ou indica ou chanvre indien (Bangh, Gunjah, Gna\%a), orjginaire des Indes, y est propagée comme en Perse et en Arabie. $\Lambda$ u point de vne de la classification botanique, elle ne diffère pas sensiblement de notre variété européenne, mais elle s'en distingne d'une manière alssolue par des éléments constituants qui sont particnlièrement actifs.

\section{Préparations et principes.}

Nous ne parlerons pas des préparations informes et dangerense qui sont commes sous le nom de haschich, et qui contiennent sonvent de l'opium, quelquefois des cantharides, toujours 111 poison tétanisant des plus violents, qui a été indiqué par l'ersonne, en 1857, étudié par $\mathrm{Hay}$, en $\mathbf{1 8 8 3}$, et qui se rapproche singulierement de la strychuine ou bien encore de la thebaine contenue dans l'opium. La description du haschich, pour ceux qui s'y intéressent, se tronve dans $1 m$ récent et excellent article de M. Egasse (Bulletin de Thérapeutique).

La matière médicale proprenıent dite du camabis comprenait jusqu'ici deux préparations, des extraits et des teintures. Dans ces derniers temps, on a expérimenté trois principes essentiels tirés de la plante, apres cliverses manipulations.

\footnotetext{
1) Journal of Physiology. Vol. 1, p. 370, 1878-1879.

2) Philosophical 'Transactions 1890.

3) Brit. Ned. Journ., January 4 th 1890
}

\section{Frster Theil.}

Pharmakologie.

Wir theilen diese Arbeit in drei Abschnitte. Der erste beschäftigt sich mit der Pharmakologie der Cannabis, der zweite mit einer allgemeinen Untersuchung über die Neurosen uud gastrischen Dyspepsieen, der dritte mit der $\Lambda$ nwendung der Cannabis in den verschiedenen Fällen.

Die Cannabis sativa (Urticaceen) ist eine aus Westindien stammencle Pflanze, die in Europa ihrer sehr ölhaltigen Körner wegen cnltivirt wird. Eine Varietät dieser Pflanze, die Cannabis sativa indica oder indischer Hanf (Bheng, Gunjalı, Guaza), ist in Indien „u Hause und hat sich auch in Persien und. Arabien verbreitet. Hinsichtlich ihrer botanischen Classificirnng unterscheidet sie sich nicht merkbar von unserer europäischen Varietät, dagegen in absolnter Weise durch ihre wirksamen Stoffe.

\section{Präparate und Bestandtheile.}

Wir sprechen hier niclit von den ganz inconstant zusanmengesetzten nnd gefälnlichen Präparaten, bekannt unter dem Nameu Hasehisch, die oftmals Opium, manchmal auch Canthariden enthalten, und die stets eins der heftigsten tetanisirenden Gifte sind, auf das 1857 Personne zuerst aufmerksam gemacht, das Hay 1883 näher untersucht hat, und das dem Strychnin und auch wohl dem im Opinm enthaltenen Thebaïn ausserordentlich mahesteht. Die Beschreibung des Haschisch findet sich für diejenigen, welche sich dafür interessiren, in einer kürzlich veröffentlichten vortrefflichen Arbeit von M. Egasse (Bulletin de Thérapeutiqne)

Pharmaceutische Prïparate der Cannabis im eigentlichen Sinuc gab es bisher zwei, das Extract und die 'Tinctur. In neuerer Zeit hat man mit drei nach verschiedenartigem Verfahren ans der Pflanze gewonuenen wesentlichen Bestaudtheilen Versuche angestellt.

1) Journal of Physiology. Vol. I, p. 370, 1878-1879.

2) Philosophical Transactions. 1890.

3) Brit. Mled. Journ., 4. Jantant 1890. 
Composition de la plante. La plante elle-même contient principalement:

1. Cannabinine. Un alcaloïde volatil mal caractérisé et désigné par Siébold et Bradbury, sous le nom de cannabinine. Est-ce l'analogue d'une cannabine, d'un glycoside récemment employé.

2. Cannabinon. Une masse amorphe, résineuse, amère, décrite par Martin, en 1855, qui a été proposée et employée depuis quelque temps, sous le nom de cannabinon.

3. Autres principes. Personne a décrit ell outre, en 1857, une huile éthérée, d'une odeur vireuse, formée par ce qu'il appelle la cannabène. Le même auteur a décrit en outre sous le nom de haschichine, un glycoside possédant la propriété somnifère.

Toutes ces substances sont contenues, en partie au moins, dans les extraits comme dans les teintures, mais leur quantité réciproque étant variable, on a songé à isoler les produits purs que voici:

I. Tannate de cannabine. C'est une combinaison d'un glycoside appelé cannabine, mais on en ignore le mode de préparation qui est resté secret. On croit qu'il contient plusieurs glycosides, et on est certain qu'il ne contient pas la tétanine, cet alcaloïde si toxique du haschich.

Propriétés du tannate. C'est une poudre amorphe, jaunâtre ou brun gris, d'une odeur faible de chanvre, d'un gon̂t amer et astringent, qui brîle sur le platine, se dissout mal dans l'ear, l'alcool et l'éther, mais qui s'associe facilement avec l'eau acidnlée d'acide chlorhydrique à chaud, et à froid dans l'alcool acidulé de même. Quand on traite l centigramme de cette préparation par 5 centigrammes d'étlier et une goutte de perchlorure de fer, il en résulte un tannate de fer. La solution dans l'acide chlorhydrique dilué et chaud, fournit un filtrat qui précipite en blanc par les alcalis et se trouble en brun par une solution d'iode; c'est là la cannabine. Agitée avec la lessive de soude et l'éther, cette préparation donne par l'évaporation une substance d'odeur narcotique et de réaction alcaline, c'est la cannabine pure (Fischer).

Doses et usages. Le tannate s'emploie à la dose de 25 à 50 centigranumes, comme un somnifère certain; dans les isomnies simples sans causes douloureuses et physiques. Incapable de remplacer l'opium et le chloral, il est précisément à employer quand ces médicaments sont contrindiqués.

La dose maxima est de 1 gramme, la dose iournalière maxima de 2 grammes.
R. Tannate de cannabine.
1 gramme.
Sucre blanc.

2 grammes.

Divisez en 4 paquets. - 1 paquet le soir avant le coucher.

Cannabine pure. Obtenue par Bombelon par l'action de l'oxyde de zinc sur le tannate de cannabine (avec formation de tannate de zinc et de cannabine), c'est une poudre brune non adhérente, qui se consume entièrement sur le platine; insoluble dans l'eau, l'alcool, très soluble dans l'éther, le chloroforme.

Formules et doses. De 5 à 10 centigrammes, comme narcotique.

Mélangez avec la poudre de café torréfié.

II. Cannabinon. Préparation. Si l'on précipite du haschich la tétanine, à laide de l'acide tannique, on obtient une résine molle brune qu'on appelle cannabiuon. Ce produit n'a plus les propriétés excitantes du haschicl, qui sont dues à la tétanine; il possède plutôt les propriétés narcotiques.

Formules et doses. \& à 10 centigrammes (dose maxima, 10 centigrammes pour un jour).

En poudre fine mêlée avec la poudre de café, ou avec du sucre de lait, à 30 centigrammes, la dose peut devenir toxique.

Usages. C'est un somnifère préférable aux autres préparations de cannabis, il ne produit ni céplalalgie, ni constipation, et parâ̂t augmenter l'appétit.

'I'rès employée dans l'hystérie où je l'ai vue produire une réelle sédatiou, daus les affections mentales, la dose doit être augmentée pour agir:

Ou l'a proscrite, mais à tort, dans les cardiopathies, c'est le contraire que nous prouverons.

III. Extraits. On prépare anjourd'luni trois sortes d'extrajts:

1. Un extrait alcoolique fait avec de l'alcool fort (90 degrés) et lavé à l'eau. Cet extrait porte le nom de Haschichine;

2. Un extrait alcoolique fait avec l'alcool à 60 degrés désigné sons le nom d'extrait alcoolique de chanvre indien;

3. Un extrait gras que l'on préparait autrefois en faisant digérer à une douce chaleur de chanvie indien daus du beurre. Aujourd'hui, on a abandonné ce mode de préparation et l'on dissout simplement par la chaleur de la Haschichine dans du beure.
Zusammensetzung der Pflanze. - Die Pflanze selbst enthält hauptsächlich :

1. Cannabinin. Ein von Siébold und Bradbury als Cannabinin beschriebenes schlecht charakterisirtes flüchtiges Alkaloid. Es ist vielleicht gleichbedeutend mit dem Cannabin, einem kürzlich angewandten Glykosid.

2. Caunabinon. Eine amorphe, harzige Masse von bitterem Geschmacke, 1855 von Martin beschrieben, die seit einiger Zeit unter der Bezeichnung Cannabinon in Vorschlag gebracht und angewandt ist.

3. Andere Bestandtheile. Personne hat ausserdem 1857 ein ätherisches Oel von widerlichem Geruche, gebildet durch eine von ihm Can naben genannte Substanz, und ein, schlafbringende Eigenschaft besitzendes, Glykosid, unter dem Namen Haschischin, beschrieben.

Alle diese Substanzen sind, wenigstens theilweise, in dem Extracte wie in der Tinctur enthalten, doch war man, da ihre wechselseitigen Mengenverhältnisse darin schwanken, bestrebt, die reinen Producte folgendermaassen zu isoliren.

I. Cannabinum tannicum. Es ist das eine Verbindung eines Cannabin genannten Glykosid, dessen geheim gebliebene Darstellungsweise man aber nicht kennt. Mau glaubt, dass es mehrere Glykoside enthält, und weiss bestimmt, dass das Tetanin, das so stark giftige Alkaloid des Haschisch, nicht darin enthalten ist.

Eigenschaften der gèrbsauren Verbindung. Es ist ein amorphes, gelbliches oder graubräunliches Pulver, von schwachen Hanfgeruche, bitierem und adstringirendem Geschmacke, das auf Platin verbrennt, sich in Wasser, Alkohol und Aether schlecht löst, leicht aber in heissem, mit Salzsäure angesäuertem Wasser und in kaltem in gleicher Weise angesänertem Alkohol. Behandelt man $1 \mathrm{cg}$ dieses Präparates mit $5 \mathrm{cg}$ Aether und einem Tropfen Liq. Ferri sesquichlorati, so bildet sich ein Eisentinnat. Die Lösung in heisser verdünnter Salzsäure ergiebt ein Filtrat, das durch Alkalien weiss gefällt und clurch eine Jodlösung braun getrïbt wird; dies ist das Cannabin. Mit Natronlauge und Aether stark geschüttelt bildet dies Präparat durch Verdunstung eine Substanz von narkotischem Geruch und alkalischer Reaction, das reine Cannabin (Fischer).

Dosen und Anwendung. Das Tannat wird in einer Dosis von $0,25-0,5 \mathrm{~g}$ als ein sicheres Schlafmittel angewandt bei einfacher Schlaflosigkeit ohne schmerzhafte physische Ursachen. Es ist nicht imstande, das Opium und das Chloral zu ersetzen, findet aber gerade dann seine Anwendung, wenn diese Medicamente contraindjcirt sind.

Die Maximaleinzeldosis ist $1 \mathrm{~g}$, die Maximaltagesdosis $2 \mathrm{~g}$. Rp. Cannabin. tann. 1,0

Sacch. alb.

M. f p. Divid. jn part. aeql. No. IV.

S. Abends 1 Plvr. vor dem Schlafengeheu zu nehmen.

Cannabinum purum. Bombelon gewann es durch Einwirkung von Zincum oxydatum auf das Cannabinum tannicum (unter Bildung von Zincum tannicum und Cannabin). Es ist ein braunes, lockeres Pulver, das anf Platin ohne Rückstand verbrennt; unlöslich in Wasser und Alkobol, leicht löslich in Aether und Chloroform.

Formeln nnd Dosen. 0,05-0,1 g als Narkoticum, mit gemallenem geröstetem Kaffee zu vermischen.

II. Cannabinon. Darstellung. Fällt man aus dem Haschisch durch Acidum tannicum das Tetanin aus, so erlaält man ein weiches braunes Harz, das Cannabinon genannt wird. Dies Product hat nicht mehr die erregenden Eigenschaften des Haschisch, die auf dem Tetanin beruhen, sondern wirkt vielmehr als Narkoticum.

Formeln und Dosen. ad 0,03 pr. dosi! ad 0,1 pro die!

Als feines Pulver mit gemahlenem Kaffee oder Milchzucker, bis zu 0,3 , doch kann diese Dosis toxisch wirken.

Anwendung. Als Schlafmittel den anderen Cannabispräparaten vorzuziehen; es orzeugt weder Kopfschmerz, noch Stuhlverstopfung und scheint den Appetit zu befördern.

In der Hysterie, wo ich es eine unzweifelhafte Beruhigung hervorrufen sah, und bei Geisteskrankheiten viel angewandt, wo die Dosis, um wirksam zu sein, stark vergrössert werden muss.

Man verordnet es auch, aber mit Unrecht, bei Herzleiden; hier jst es, wie wir nacliweisen. werden, geradezu contraindicirt.

III. Extracte. Man bereitet \%ur Zeit drei Arten von Extracten:

1. Ein alkoholisches Extract, mit starkem Alkohol $\left(90^{\circ}\right)$ bereitet und mit Wasser ausgewaschen. Dies Extract führt den Namen Haschischin.

2. Ein alkoholisehes Extract, mit 60 gradigem Alkohol hergestellt und als alkoholisches Extract aus indischem Hanf bezeichnet.

3. Ein fettes Extract, das man sonst dadurch herstellte, dass man indischen Hanf bei gelinder Hitze in Butter digeriren liess. Jetzt ist diese Darstellungsweise aufgegeben, und man löst einfach auf dem Fener Haschischin in Butter auf. 
La préparation la plus énergique est. la $\mathrm{Haschichine,} \mathrm{car} \mathrm{cet}$ extrait est préparé à l'alcool à 90 degrés et de plus repris par l'eau qui enlève toutes les substances solubles dans ce véhicule.

D'après M. Dausse, voici l'ordre de puissance de ces différents produits. Si l'on représente par 100 la valeur de la haschichine, on a le tableau suivant:

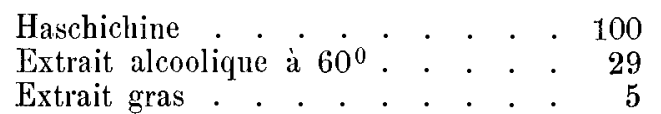

L'extrait gras ne contient donc que $1-20$ de haschichine.

\section{Expérimentation des nouvelles préparations de cannabis.}

Ce sont les trois préparations nouvelles qui ont été recommandées pour remplacer le cannabis indica:

Le tannate de cannabine:

Le cannabinon;

Le baume de cannabis.

Prior (Münch. med. Wochschr. 1888) les a expérimentées sur un grand nombre de malades (phtisiques, emphysémateux, agrypniques, vieillards avec agitation).

Tannate de cannabine Dose employée: 0,50 à 1,25.

Sur 35 individus qui prirent 100 doses de tannate, Prior constata dans 42 expériences, un excellent effet; 17 fois un effet incomplet, 40 fois nul et une fois détestable dans un cas de delirium trémens; dans les cas efficaces, il se manifesta après une demiheure à 1 henre une sensation de somnolence et de fatigue, suivic d'un sommeil de 5 à 6 heures.

Canuabin on en tablettes de chocolat contenant chacune 0,06 de canuabinon (B ombelon). Des essais furent pratiqués che $z 18$ femmes (dont me prit 0.10 de cannabinon) et che 8 hommes. Chez tous le remède provoqua de la céphalalgie, des vomissements, de l'exaltation.

Baume de cannabis, recommandé par Denzel à la dose de 0,10 à 0,30 comme inoffensif. Prior l'employa de même et poussa la dose à 0,20 chez un emphysémateux qui ne tarda pas à éprouver des tronbles céphaliques ot digestifs. Chez 3 cardiaques les effets furent mauvais.

Les deux dernières préparations sont peu recommandables, principalement chez les cardiaques.

Doses toxiques de cannabinon. Un cas personnel de Pusinelli (de Dresde). Ce médecin prit 0,06 (en tablette); au bout d'une demi-heure il ne parvint plus à construire les phrases; puis il eut une excitation comme électrique, avec agitation croissante; mais la connaissance était si bien conservée que le patient put saisir toutes les nuances de son mal; au bout de 4 heures il était guéri. (Deut. med. Woch., 1886, no 46 ).

Doses toxiques des extraits et teintures. De nombreuses plaintes zur la toxicité ou sur la nullité des préparations de cannabis s'élevèrent de toutes parts, comme les récriminations s'adressaient jusqu'à hier à la variabilité des préparations de digitale. En 1887, lo Dr. Schuschny (de Buda-Pest) apprit d'un confrère qui donna 1 gramme d'extrait de cannabis pour 150 grammes d'émulsion, par cuillerées toutes les deux heures à mue vieille femme atteinte de spasme vésical, qu'après mne dose 0,25 , la malade éprouva une grande angoisse sans dyspnée r'élle, avec' un vertige immense, le pouls à 132; des sueurs profuses, les pupilles dilatées, et avec tont cela parfaite conservation de l'intelligence. Guérison au bout de 8 heures.

'l'einture. 20 gouttes avec 70 centigrammes de chloral et 2 grammes de bromure ont été prescrites par Mac Gonnell (Practitioner 1888) comme hypnotique dans les maladies du cœur et chez les brightiques lorsque l'opinm était contrindiqué.

On ne coniprend pas cette mixture, pas plus que le bromidia qui renferme me deni-douzaine de substances. La teinture sans mélange a été aussi employée par J. William $0^{\prime} S$ changulessy vers 1840, comme apéritif et digestif; nous nous en sommes souvenu.

Rnssel (1890) vient de préconiser la teinture dans une foule de cas les plus divers, tels que la manie aiguë, la chorée, etc, que je n'ai pas pu éclaircir. Il est un point sur lequel nons sommes d'accord, c'est sur la dose, qui est exactement la même dans mes recherches qui datent d'un an, et dans les observations plus récentes de Russel. Il fixe, comme nous, la dose de teinture à l'équivalence d'un grain d'extrait, c'est-à-dire, 0,05 d'extrait gras par jour. Toutes nos observations ont été faites à l'aide de cet extrait, les principes chimiques purs nous ayaut mauqué ou ayant manqué leurs effets.
Das kräftigste Präparat ist das Haschischin, denn dies Extract wird mit 90 gradigem Spiritus bereitet und ausserdem mit Wasser ausgewaschen, das alle in diesem Vehikel löslichen Substanzen entfernt.

Nach M. Dausse ist der Wirksamkeitsrang dieser verschiedenen Producte folgender: Bezeichnet man den Werth des Haschischin mit 100 , so hat man folgende Tabelle:

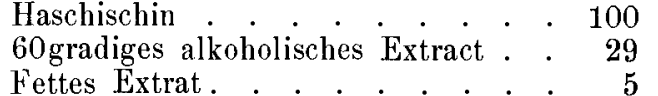

Das fette Extract enthält somit nur 1/20 Haschischiı.

\section{Versuche mit neuen Cannabispräparaten}

Es sind diei neue Präparate zum Ersatze der Cannabis indica empfohlen worden:

Das Cannabinum tannicum;

Das Cannabinon;

Der Cannabisbalsam.

Prior (Münch. med. Wochenschrift 1888) hat sie an eiuer grossen Zahl von Kranken (Phthisikern, Emphysematikern, Schlaflosen, Greisen mit starker Unruhe) versucht

Cannabinum tannicum. Angewandte Dosis: 0,5 bis 1,25. - An 35 Individuen, die 100 Dosen des Tannates nahmen, constatirte Prior in 42 Versuchen eine vorzügliche Wirkung, $17 \mathrm{mal}$ eine unvollständige Wirkung, während $40 \mathrm{mal}$ die Wirkung gann/ ausblieb, und in einem Falle von Delirium tremens dieselbe geradezu schädlich war. In den wirksamen Fällen stellte sich nach einer halben bis einer Stunde ein Gefühl von Schläfrigkeit und Ermattung ein, dem ein fünf- bis sechsstündiger Schlaf folgte.

Cannabinon in Chocoladenplättchen mit einem Gehalte von 0,06 Cannabinon in jedem (Bombelon). - Versuche wurden al 18 Frauen (von deuten eine 0,1 Cannabinon nahm) und an 8 Mäınern angestellt. Bei allen rief das Mittel Kopfschmerzen, Erbrechen, Aufregung hervor.

Cannabisbalsam, von Denzel in einer Dosis von 0,1 bis 0,3 als unschädlich empfohlen. Prior wandte es in gleicher Stärke an und stieg bei einem Emphysematiker mit der Dosis auf 0,2 , wonach sich bald Gehirn- und Verdauungsstörungen einstellten. Bei drei Herzkranken waren die Wirkungen nachtheilig.

Die beiden letzteren Präparate sind wenig empfehlenswerth, vornehmlich bei Herzkranken.

Toxische Dosen des Cannabinon. Ein an sich selbst beobachteter Fall von Pusinelli (Dresden). Dieser Arzt nahm 0,06 (in Plättchenform); nach Verlauf einer halben Stunde konnte er keine Sätze mebr construiren; dann empfand er eine wie elektrische Aufregung mit wachsender Unruhe. Aber das Bewasstsein war so wohl erhalten, dass der Patient alle feinen Abstufingen seines krankhaften Zustandes aufzufassen vermochte; nach Verlauf von vier Stunden Heilung. (Deutsche med. Wochenschrift 1886, Nr. 46.)

Toxische Dosen der Extracte und Tiucturen. Zahlreiche Klagen über die Giftigkeit oder die Wirkungslosigkeit der Cannabispräparate wurden von alleu Seiten laut, wie auch noch bis vor kurzem über die Veränderlichkeit der Digitalispräparate. Im Jalıre 1887 erfuhr Dr. Schuschn y (Budapest) von einem Collegen, der 1 g Extract. Cannabis anf $150 \mathrm{~g}$ Emulsion 2 stündlich einen Löffel voll einer an Blasenkrampf leidenden alten Frau gab, dass die Patientin nach einer Dosis von $0,25 \mathrm{~g}$ ein starkes Angstgefühl ohne wirkliche Dysunoe mit heftigem Schwindel empfand, Puls 132; profuse Schweisse, dilatirte Pupillen, und dabei vollständige Erhaltung des Bewusstseins. Heilung nach Verlanf von 8 Stunden.

Tinctur. 20 Tropfen mit 0,7 Chloral und 2,0 Bromkalium sind von Ma c Counell (Practitioner, 1888) als Hypnoticum bei Herzkrankheiten und Morbus Briglitii verordnet worden, wenn Opinm contraindicirt war:

Diese Mixtur ist unverständlich, ebenso wie die Brommixtur mit ihren halbdutzend Substanzen. Die unvermischte 'Tinctur ist ebenfalls, und zwar von J. William O'Schangnessy um das Jahr 1840 als Aperitivum und Digestivum angewandt worden; wir entsinnen uns dessell.

Russel (1890) hat ganz kürzlich die Tinctur in einer Menge ganz verschiedenartiger Fülle, wie in der acuten Manie, in der Chorea u. s. w., gerïhnut, worüber ich mir keine Aufklärung habe verschaffen können, In einem Punkte sind wir völlig e:ner $\Lambda_{11-}$ sicht, nämlich iı Betreff der Dosis, die nach meinen ein Jahr alten Untersuchungen wie nach den neueren Beobachtungen von Russel genau die gleiche ist. Er fixirt, wie wir, die Dosis der Tinctur gleich einem Gran Extract, d. l. 0,05 fetten Extractes pro die. Alle unsere Beobachtungen siud mit Hülfe dieses Extractes angestellt worden, da die rein chemischen Stoffe uns theils gefehlt, theils ilıre Wirkung versagt hatten. 


\section{Expériences nouvelles avec les extraits.}

La mullité des effets obtenus par les principes chimiques fut pour moi le motif décisif de leur abandon. Mais comme dans le cours d'études récentes pliysiologiques et cliniques sur les somnifères je fus amené à la recherche comparative des propriétés soporifiques tant variées du cannabis, il me fallut expérimenter d'abord avec l'extrait qu'on appelle aussi extrait gras, n'ayant pu obtenir jusqu'ici, malgré plusieurs travaux analytiques, le véritable principe artif alssolument isolé. Grande fut ma surprise quand, après de nombreuses tentatives, je constatai la parfaite nullité de l'extrait de cammabis comme soporifique et surtout comme paralgésiant; il n'a rien de commun avec le chloral d'ancienne date, ni avec le chloramide de date récente dont j'ai vérifié les effets somnifères; il n'a rien de conmun avec la morphine ou la codéine ni avec l'antipyrine et les névrotiques de ce genre qui agissent si puissament pour supprimir la douleur; ce qui me frappa c'est le pouvoir sédatif, qui se retrouve en grande partie dans le bromure de potassium, mais avec cette différence que le bromure agit sur le centre cérébrospinal, tandis que le cannabis agit sûrement sur le système nerveux de l'estomac, et presque certainement sur celui du cour; au premier point de vue on pent dire que c'est un vrai sédatif gastro-intestinal.

Dose et mode d'employ. Après de longues hésitations et des tâtonmentents nombreux, je suis arrivé à fixer la dose de l'extrait à 5 centigrammes par jour, dose qui doit d'ailleurs varier selon la nervosité des malades. Avec cette indication, il n'y a rien ì craindre des effets du médicament; si on dépasse la dose de cing centigrammes par jour, on observe des effets singuliers, mais tout ¿̀ fait passagers d'ébriété; à partir de 10 centigranmes par jour ces accidents sont certains. Avec la quantité indiquée pour l'adulte lomme ou femme, je n'ai jamais observé le moindre inconvénient i signaler; l'action médicamenteuse est pour ainsi dire immédiate sur l'estomac à l'état pathologique.

Avant de poser les indications du médicament et de préciser ses usages, il finut naturellement faire une étude sommaire des affections morljides qui relèvent de cette médication; ce sont surtout les dyspepsies chiniques et les névroses; la deuxième partie de notre travail est destinée à les synthétiser d'abord, à les comparer ensuite, et chemin faisant, à en expliquer le mode de formation; ce n'est qu'après cette cuvre préliminaire et aussi alorégée que possible, après ce parallèle qui n'a pas encore été tenté, que nous pourrons saisir les occasions et les raisons du traitement en partie nouveall.

\section{Neue Experimente mit den Extracten.}

Die bei Auwendung der chemischen Grundstoffe beobaclitete Wirkungslosigkeit war für mich das entscheidende Motiv, von jenen Abstand zu nehmen. Da ich aber im Verlaufe neuerer physiologischer und klinischer Studien über die Somnifera zur vergleichenden Untersuchung der so maunigfaltigen, schlafbringenden Eigenschaften der Cannabis geführt wurde, so musste ich zuerst mit dem Extract, auch fettes Extract genannt, experimentiren, da ich bis jetzt trotz mehrfacher analytischer Arbeiten den wirklichen wirksamen Grundstoff nicht vollständig isolirt herzustellen vermochte. Gross war meine Ueberraschung, als ich nach zahlreichen Versuchen die vollstäıdige Wirkungslosigkeit des Cannabisextractes als Schlafmittel und vornehmlich als schmerzstillendes Mittel feststellte. Es hat nichts gemein mit dem Chloral alten Datums, noch mit dem Chloralanid nenen Datums, dessen schlafbringende Wirkungen ich bestätigt habe; es hat nichts gemein mit dem Morphium oder dem Codeîn, nichts mit dem Antipyrin und den Neuroticis dieser Gattung, die eine so mächtige schmerzbeseitigende Wirkung haben. Was mir auffiel, war das beruhigende Vermögen, wie es sich zum grossen Theile im Bromkalium wiederfindet, nur mit dem Unterschiede, dass das letytere auf das Cerebrospinalcentrum einwirkt, die Cannabis dagegen absolut sicher auf das Nervensystem des Magens und fast sicher auf das des Herzens. In ersterer Hinsicht kann mau sagen, es sei ein wahres gastro-intestinales Sedativum.

Dosis und Anwendungsweise. Nach langen Schwanken und zahlreichen Versuchen gelangte ich dazu, die Dosis des Extractes auf 0,05 pro die festzusetzen, eine Dosis, die übrisens je nach der Nervosität der Kranken variirt werden muss. Mit dieser Indication braucht man in Betreff der Wirkungen des Medicamentes keine Befuirchtungen iu hegen. Ueberschreitet man die Dosis von 0,05 pro die, so beobachtet man eigenthürnliche, aber durchaus rasch vorïbergehende 'Trunkenheitserscheinungen; von $0,1 \mathrm{~g}$ pro die au treten solche Zufảlle sicher ein. Bei der angegebenen Dosis für Erwachsene habe ich njemals die geringste Unzuträglichkeit zu verzeichnen gehabt. Die medicamentöse Wirkung ist so zu sagen eine unmittelhare auf den kranken Magen.

Vor Feststellung der Indicationen des Medicamentes und Präcisirung seiner Anwendung ist naturgemäss erst eine summarische Abhandlung über die krankhaften Zustände nothwendig, welche durch diese Medication gelıoben werden. Es sind dies vornehmlich die chemischen Dyspepsieen und die Neurosen. Der zweite Theil unserer Arbeit beschäftigt sich zuerst mit ihrer Synthese, dann mit ilırer Veroleichung und im weiteren Verlanfe mit der Erklärung ihrer Bildungsweise. Erst nach dieser möglichst kurzen PrälimiuarArbeit, nach dieser Parallele, die bisher noch gar nicht versuch worden ist, können wir uns üher das Wann nnd das Wie der zum Theil neuen Behandlungsweise verständigen. 